\title{
Prediction of Times to Failure of Censored Units in Hybrid Censored Samples from Exponential Distribution
}

\author{
A. Asgharzadeh ${ }^{\dagger, *}$ and R. Valiollahi ${ }^{\ddagger}$ \\ ${ }^{\dagger}$ University of Mazandarann \\ ¥ Semnan University
}

\begin{abstract}
In this paper, we discuss different predictors of times to failure of units censored in a hybrid censored sample from exponential distribution. Bayesian and non-Bayesian point predictors for the times to failure of units are obtained. Non-Bayesian prediction intervals are obtained based on pivotal and highest conditional density methods. Bayesian prediction intervals are also proposed. One real data set has been analyzed to illustrate all the prediction methods. Finally, different prediction methods have been compared using Monte Carlo simulations.
\end{abstract}

Keywords. Hybrid censoring; exponential distribution; maximum likelihood predictor; best unbiased predictor; conditional median predictor; Bayesian predictor; Monte Carlo simulation.

MSC 2010: 62N01, 62M20, 62F15.

\section{Introduction}

The two most common censoring schemes are termed as Type-I and TypeII censoring schemes. Consider a sample of $n$ units placed on a lifetime experiment at time 0. In Type-I censoring scheme, the lifetime experiment is terminated when a pre-fixed censoring time $T$ arrives. In Type-II censoring scheme, the experiment may instead be terminated when the $r$ th $(r \leqslant n$ is

* Corresponding author 
fixed) failure is observed. Hybrid censoring scheme is a mixture of TypeI and Type-II censoring schemes. In this censoring scheme, the lifetime experiment terminates as soon as either the $r$ th $(r \leqslant n$ is fixed) failure or the pre-determined censoring time $T$ occurs. Thus, in this censoring scheme one observes $x_{1: n}, \ldots, x_{d: n}$ when $x_{d: n} \leqslant \min \left\{x_{r: n}, T\right\}, d \leqslant r$ and $x_{d+1: n}>\min \left\{x_{r: n}, T\right\}$. Here $x_{1: n}<x_{2: n} \cdots<x_{n: n}$ denote the observed ordered failure times of $n$ units. It is clear that Type-I and Type-II censoring schemes can be obtained as special cases of hybrid censoring scheme by taking $r=n$ and $T=\infty$, respectively.

Epstein (1954) introduced hybrid censoring scheme, and considered lifetime experiments assuming that the lifetime of each unit follows an exponential distribution. In the recent years, many authors have discussed statistical inference problems for various distributions under hybrid censoring scheme. Gupta and Kundu (1998) obtained confidence and credible intervals for the exponential mean lifetime $\theta$. Based on the distribution of the Maximum likelihood estimator (MLE), Chen and Bhattacharya (1988) obtained exact confidence intervals for the exponential parameter $\theta$. Later, using gamma prior distribution, Draper and Guttman (1987) obtained Bayes estimates and credible intervals for $\theta$. Recently, Kundu (2007) obtained the MLEs, the approximate MLEs and Bayes estimates of shape and scale parameters of a Weibull distribution. We also refer to Fairbanks et al. (1982), Draper and Guttman (1987), Ebrahimi (1986, 1992), Jeong et al. (1996), Childs et al. (2003), Kundu and Banerjee (2008) and Kundu and Howlader (2010) for some more work under hybrid censoring.

Prediction of future failures given a record of observed failures is an interesting topic, especially in medical and engineering sciences. Information regarding future observations can tell us at an early stage of testing how costly the testing is and whether actions should be taken to redesign the test. Extensive work on prediction problem can be found in the literature. Lawless (1971) derived prediction intervals for future failures under Type-II censoring scheme. Dunsmore (1983) discussed the prediction of the future records through tolerance regions and Bayesian predictive distributions. Kaminsky and Rhodin (1985) applied the principle of maximum likelihood to the joint prediction and estimation of a future random variable and an unknown parameter. An excellent review of development on prediction problems can be found in Kaminsky and Nelson (1998). See also the work of Awad and Raqab (2000), Basak et al. (2006) and Ren et al. (2006).

Let $\mathbf{X}=\left(X_{1: n}, \ldots, X_{d: n}\right)$ denote a hybrid censored sample from an ex- 
ponential model. The aim of this paper is to discuss the prediction of the future failures times $Y=X_{s+d: n}(s=1,2, \ldots, n-d)$ based on observed data $\mathbf{x}=\left(x_{1: n}, \ldots, x_{d: n}\right)$. We study this problem via non-Bayesian and Bayesian approaches and present several predictors of $Y=X_{s+d: n}(s=1,2, \ldots, n-d)$.

Based on the observed hybrid sample $\mathbf{x}=\left(x_{1: n}, \ldots, x_{d: n}\right)$, Ebrahimi (1992) discussed the maximum likelihood predictor (MLP) of $Y$. The main difference of our work with the existing work (Ebrahimi, 1992) is that our proposed methods are quite general. We have considered different classical point and interval predictors which have not been considered before. Moreover, we have also used Bayesian approach to compute the predictive density and also to compute the corresponding Bayesian interval.

Rest of the paper is organized as follows. Section 2 contains some preliminaries. The different point predictors are considered in Section 3 under non-Bayesian and Bayesian approaches. In Section 4, we provide three types of prediction intervals (PI's) for $Y=X_{s+d: n}(s=1,2, \ldots, n-d)$. One real data set has been analyzed in Section 5. Section 6 includes an extensive simulation study to illustrate all the prediction methods and a discussion of the results. Finally in Section 7, we conclude the paper.

\section{Preliminaries}

Let $\mathbf{X}=\left(X_{1: n}, \ldots, X_{d: n}\right)$ denote a hybrid censored sample from an exponential distribution (denoted by $\exp (\theta)$ ) with the density function

$$
f(x ; \theta)=\theta e^{-\theta x}, \quad x>0, \quad \theta>0 .
$$

For notation simplicity, we will write $\left(X_{1}, X_{2}, \ldots, X_{d}\right)$ for $\left(X_{1: n}, X_{2: n}, \ldots, X_{d: n}\right)$. Based on the observed data, the likelihood function for $\theta$ without the normalizing constant is given by

$$
L(\theta)=\theta^{d} e^{-\theta\left[\sum_{i=1}^{d} x_{i}+(n-d) T_{0}\right]}
$$

where $d$ denotes the number of failures and $T_{0}=\min \left\{x_{r: n}, T\right\}$. From (2), the maximum likelihood estimator (MLE) of $\theta$ is derived to be

$$
\widehat{\theta}=\frac{d}{\sum_{i=1}^{d} x_{i}+(n-d) T_{0}} .
$$

Due to the Markovian property of censored-order statistics, it is wellknown that the conditional density of $Y=X_{s+d: n}$ given $\mathbf{X}=\mathbf{x}$ is the same 
as the density of the $s$ th order statistic out of $n-d$ units from the population with density $f(y) /\left(1-F\left(T_{0}\right)\right), y \geqslant T_{0}$ (left truncated density at $T_{0}$ ). Therefore, the conditional density of $Y=X_{s+d: n}$ given $\mathbf{X}=\mathbf{x}$, for $y \geqslant T_{0}$, is given by

$$
f(y \mid \mathbf{x})=s\left(\begin{array}{c}
n-d \\
s
\end{array}\right) f(y)\left[F(y)-F\left(T_{0}\right)\right]^{s-1}[1-F(y)]^{n-d-s}\left[1-F\left(T_{0}\right)\right]^{-(n-d)} .
$$

For model (1), (4) reduces to

$$
f(y \mid \mathbf{x}, \theta)=s\left(\begin{array}{c}
n-d \\
s
\end{array}\right) \theta e^{-\theta\left[(n-d-s+1) y-(n-d) T_{0}\right]}\left[e^{-\theta T_{0}}-e^{-\theta y}\right]^{s-1}, \quad y \geqslant T_{0} .
$$

The predictive likelihood function (PLF) of $Y$ and $\theta$, is given by

$$
L(y, \theta ; \mathbf{x})=f(y \mid \mathbf{x} ; \theta) f(\mathbf{x} ; \theta) .
$$

Consequently, for the exponential model (1), the PLF of $Y$ and $\theta$ can be obtained as

$$
\begin{aligned}
L(y, \theta)= & s\left(\begin{array}{c}
n-d \\
s
\end{array}\right) \theta^{d+1}\left[e^{-\theta T_{0}}-e^{-\theta y}\right]^{s-1} \\
& \times \exp \left[-\theta\left\{\sum_{i=1}^{d} x_{i}+(n-d-s+1) y\right\}\right] .
\end{aligned}
$$

By differentiating the predictive log-likelihood function $\ln L(y, \theta)$ with respect to $y$ and $\theta$, respectively, and equating them to zero, one can obtain the maximum likelihood predictor (MLP) of $Y$ and the predictive maximum likelihood estimator (PMLE) of $\theta$ as (see Ebrahimi, 1992)

$$
\widehat{Y}_{M L P}=T_{0}+\ln \left(\frac{n-d}{n-d-s+1}\right) \frac{\sum_{i=1}^{d} x_{i}+(n-d) T_{0}}{d+1},
$$

and

$$
\widehat{\theta}_{P M L E}=\frac{d+1}{\sum_{i=1}^{d} x_{i}+(n-d) T_{0}} .
$$




\section{Point Predictors}

In this section, based on the observed hybrid sample $\mathbf{x}=\left(x_{1}, \ldots, x_{d}\right)$, we provide several point predictors of $Y=X_{s+d: n}$ using non-Bayesian and Bayesian approaches.

\subsection{Non-Bayesian Predictors}

The maximum likelihood predictor is a non-Bayesian predictor which is obtained using the likelihood prediction method. In this subsection, we use the conditional prediction method to obtain two conditional predictors namely best unbiased predictor (BUP) and conditional median predictor (CMP).

A statistic $\hat{Y}=\delta(\mathbf{x})$ which is used to predict $Y=X_{s+d: n}$ is said to be a predictor of $Y$. The predictor $\hat{Y}$ is a BUP of $Y$, if the predictor error $\hat{Y}-Y$ has a mean zero and its prediction error variance $\operatorname{var}(\hat{Y}-Y)$ is less than or equal to that of any other unbiased predictor of $Y$.

In view of (5), the BUP of $Y$ is

$$
\hat{Y}_{B U P}=E(Y \mid \mathbf{X})=\int_{T_{0}}^{\infty} y f(y \mid \mathbf{x}, \theta) d y .
$$

Substituting $f(y \mid \mathbf{x}, \theta)$ and using the binomial expansion

$$
\left[e^{-\theta T_{0}}-e^{-\theta y}\right]^{s-1}=\sum_{i=0}^{s-1}\left(\begin{array}{c}
s-1 \\
i
\end{array}\right)(-1)^{s-i-1} e^{-\theta i T_{0}} e^{-\theta(s-i-1) y}
$$

we obtain the BUP as

$$
\hat{Y}_{B U P}=\frac{s}{\theta}\left(\begin{array}{c}
n-d \\
s
\end{array}\right) \sum_{i=0}^{s-1}\left(\begin{array}{c}
s-1 \\
i
\end{array}\right)(-1)^{s-i-1} \frac{\theta(n-d-i) T_{0}+1}{[n-d-i]^{2}} .
$$

When the parameter $\theta$ is unknown, it has to be estimated. Thus in this case, one would replace it by its MLE in (3) and obtain the BUP of $Y$.

Conditional median predictor (CMP) is another conditional predictor which is suggested by Raqab and Nagaraja (1995). A predictor $\hat{Y}$ is called the CMP of $Y$, if it is the median of the conditional distribution of $Y$ given $\mathbf{X}=\mathbf{x}$, that is

$$
P_{\theta}(Y \leqslant \hat{Y} \mid \mathbf{X}=\mathbf{x})=P_{\theta}(Y \geqslant \hat{Y} \mid \mathbf{X}=\mathbf{x}) .
$$


Using the relation

$$
P_{\theta}(Y \leqslant \widehat{Y} \mid \mathbf{X}=\mathbf{x})=P_{\theta}\left(1-\frac{e^{-\theta Y}}{e^{-\theta T_{0}}} \leqslant 1-\frac{e^{-\theta \widehat{Y}}}{e^{-\theta T_{0}}} \mid \mathbf{X}=\mathbf{x}\right)
$$

and using the fact that the distribution of $1-\frac{e^{-\theta Y}}{e^{-\theta T_{0}}}$ given $\mathbf{X}=\mathbf{x}$ is a $\operatorname{Beta}(s, n-d-s+1)$ distribution, we obtain the CMP of $Y$ as

$$
\hat{Y}_{C M P}=T_{0}-\frac{1}{\theta} \ln ([1-\operatorname{Med}(B)]),
$$

where $B$ has $\operatorname{Beta}(s, n-d-s+1)$ distribution and $\operatorname{Med}(B)$ stands for median of $B$. When $\theta$ is unknown, we can substitute $\theta$ with its MLE and obtain the CMP of $Y$.

\subsection{Bayesian Predictors}

In this section, our interest is to predict $Y=X_{s+d}(s=1,2, \ldots, n-d)$ based on the observed hybrid censored sample $\mathbf{x}=\left(x_{1}, \ldots, x_{d}\right)$ from a Bayesian approach.

Under the assumption that the parameter $\theta$ is unknown, we consider the gamma prior gamma $(a, b)$, with the density function

$$
\pi(\theta)=\frac{b^{a}}{\Gamma(a)} \theta^{a-1} e^{-b \theta}, \quad \theta>0, \quad(a>0, b>0),
$$

for $\theta$. When $a=b=0$, it is the non-informative prior of $\theta$. The posterior density function of $\theta$ given the data can be written as

$$
\pi(\theta \mid \boldsymbol{x}) \propto \theta^{a+d-1} e^{-\theta\left[\sum_{i=1}^{d} x_{i}+(n-d) T_{0}+b\right]},
$$

which is also gamma $\left(a+d, \sum_{i=1}^{d} x_{i}+(n-d) T_{0}+b\right)$.

The Bayes predictive density function of $Y$ given $\mathbf{x}$ is given by

$$
f_{s}^{*}(y \mid \mathbf{x})=\int_{0}^{\infty} f(y \mid \mathbf{x}, \theta) \pi(\theta \mid \mathbf{x}) d \theta .
$$

Combining the posterior density function and the conditional density given in (5), and using the binomial expansion, we obtain the predictive 
density function of $Y$ as

$$
\begin{aligned}
f_{s}^{*}(y \mid \mathbf{x})= & s\left(\begin{array}{c}
n-d \\
s
\end{array}\right) \frac{a+d}{\sum_{i=1}^{d} x_{i}+(n-d) T_{0}+b} \sum_{i=0}^{s-1}\left(\begin{array}{c}
s-1 \\
i
\end{array}\right)(-1)^{s-i-1} \\
& \times\left\{\frac{\sum_{i=1}^{d} x_{i}+(n-d) T_{0}+b}{(n-d-i)\left[y-T_{0}\right]+\sum_{i=1}^{d} x_{i}+(n-d) T_{0}+b}\right\}^{a+d+1} .
\end{aligned}
$$

Bayesian point predictors can be obtained from the predictive density function $f_{s}^{*}(y \mid \mathbf{x})$ and given the loss function. The Bayesian point predictor of $Y$ under a squared error loss, $\widehat{Y}_{S E P}$, is

$$
\widehat{Y}_{S E P}=\int_{T_{0}}^{\infty} y f_{s}^{*}\left(y \mid x_{d}\right) d y .
$$

Using (15) and after some algebra, we obtain the Bayesian point predictor $\widehat{Y}_{S E P}$ as

$$
\begin{aligned}
\widehat{Y}_{S E P}= & s\left(\begin{array}{c}
n-d \\
s
\end{array}\right) \sum_{i=0}^{s-1}\left(\begin{array}{c}
s-1 \\
i
\end{array}\right)(-1)^{s-i-1} \frac{1}{(n-d-i)^{2}} \\
& \times\left[\frac{a+d}{a+d-1}\left\{\sum_{i=1}^{d} x_{i}+(n-d) T_{0}+b\right\}-\left(\sum_{i=1}^{d} x_{i}+i T_{0}+b\right)\right] .
\end{aligned}
$$

\section{Prediction Intervals}

In this section, we consider several methods for obtaining prediction intervals (PIs) for $Y=X_{s+d}, s=(1,2, \cdots, n-d)$ based on the hybrid censored sample $\mathbf{X}=\left(X_{1}, X_{2}, \cdots, X_{d}\right)$.

\subsection{Non-Bayesian PIs}

Here we use the conditional distribution of $Y$ given $\mathbf{X}=\mathbf{x}$ to obtain two non-Bayesian PIs of $Y$. Let us fist consider the pivotal method to obtain a PI of $Y$. Consider the random variable $Z$ as

$$
Z=1-\frac{e^{-\theta Y}}{e^{-\theta T_{0}}} .
$$


As mentioned before, the conditional distribution of $Z$ given $\mathbf{X}=\mathbf{x}$ is a $\operatorname{Beta}(s, n-d-s+1)$ distribution. Thus, we can consider $Z$ as a pivotal quantity to obtain a PI for $Y$. From this, a $(1-\gamma) 100 \%$ PI for $Y$ is $\left(L_{1}(\mathbf{x}), U_{1}(\mathbf{x})\right)$ where

$$
L_{1}(\mathbf{x})=T_{0}-\frac{1}{\theta} \ln \left(\left[1-B_{\frac{\gamma}{2}}\right]\right), \quad U_{1}(\mathbf{x})=T_{0}-\frac{1}{\theta} \ln \left(\left[1-B_{1-\frac{\gamma}{2}}\right]\right),
$$

where $B_{\gamma}$ stands for $100 \gamma$ th percentile of $\operatorname{Beta}(s, n-d-s+1)$ distribution. When $\theta$ is unknown, the parameter in (17), has to be estimated. For example, by replacing $\theta$ with its MLE, the prediction limits for $Y$ can be obtained.

Another prediction interval can be obtained as follows. Since the conditional distribution of $Z$ given $\mathbf{X}=\mathbf{x}$ is a $\operatorname{Beta}(s, n-d-s+1)$ distribution with density function

$$
g(z)=\frac{z^{s-1}(1-z)^{n-d-s}}{\operatorname{Beta}(s, n-d-s+1)}, \quad 0<z<1,
$$

the $(1-\gamma) 100 \%$ highest conditional density (HCD) prediction limits for $Y$ are given by

$$
L_{2}(\mathbf{x})=T_{0}-\frac{1}{\theta} \ln \left(\left[1-w_{1}\right]\right), \quad U_{2}(\mathbf{x})=T_{0}-\frac{1}{\theta} \ln \left(\left[1-w_{2}\right]\right),
$$

where $w_{1}$ and $w_{2}$ are the simultaneous solutions of the following equations:

$$
\int_{w_{1}}^{w_{2}} g(z) d z=1-\alpha
$$

and

$$
g\left(w_{1}\right)=g\left(w_{2}\right)
$$

We can simplify Equations (19) and (20) as

$$
B_{w_{2}}(s, n-d-s+1)-B_{w_{1}}(s, n-d-s+1)=1-\alpha,
$$

and

$$
\left(\frac{1-w_{2}}{1-w_{1}}\right)^{n-d-s}=\left(\frac{w_{1}}{w_{2}}\right)^{s-1}
$$

where

$$
B_{z}(a, b)=\frac{1}{B(a, b)} \int_{0}^{z} x^{a-1}(1-x)^{b-1} d x
$$

is the incomplete beta function. 


\subsection{Bayesian PIs}

Bayesian PIs are obtained from the Bayes predictive density $f^{*}(y \mid \mathbf{x})$. Bayesian prediction bounds are obtained be evaluating

$$
P(Y>\lambda \mid \mathbf{x})=\int_{\lambda}^{\infty} f^{*}(y \mid \mathbf{x}) d y
$$

for some positive $\lambda$. Now, the $100(1-\gamma) \%$ Bayesian prediction interval for $Y$ is given by $\left(L_{3}(\mathbf{x}), U_{3}(\mathbf{x})\right)$, where the prediction limits $L_{3}(\mathbf{x})$ and $U_{3}(\mathbf{x})$ can be obtained by solving the follow nonlinear equations simultaneously

$$
P\left(Y>L_{3}(\mathbf{x}) \mid \mathbf{x}\right)=\int_{L_{3}(\mathbf{x})}^{\infty} f^{*}(y \mid \mathbf{x}) d y=1-\frac{\gamma}{2},
$$

and

$$
P\left(Y>U_{3}(\mathbf{x}) \mid \mathbf{x}\right)=\int_{U_{3}(\mathbf{x})}^{\infty} f^{*}(y \mid \mathbf{x}) d y=\frac{\gamma}{2} .
$$

Substituting $f^{*}(y \mid \mathbf{x})$ in (15) into (23) and (24), one can obtain numerically the prediction limits for $Y$.

\section{Real Data Analysis}

In this section, one real data set has been analyzed for illustrative purposes. The following ordered data represent the time to breakdown of a type of electronic insulating material subject to a constant-voltage stress:

$\begin{array}{cccccccc}0.35 & 0.59 & 0.96 & 0.99 & 1.69 & 1.97 & 2.07 & 2.58 \\ 2.71 & 2.90 & 3.67 & 3.99 & 5.35 & 13.77 & 25.50 & \end{array}$

These data are taken from Nelson (1970), and have been used earlier by Tiku and Akkaya (2004). Here, we checked the validity of the exponential distribution based on the parameter $\theta=0.4080$ using the Kolmogorov-Smirnov (K-S) test. It is observed that the K-S distance is $\mathrm{K}-\mathrm{S}=0.2315$ with a corresponding $\mathrm{p}$-value $=0.3431$. This indicates that the exponential distribution provides a good fit to the above data.

Now, suppose we use the following two sampling schemes:

$$
\begin{array}{cccc}
\text { Scheme 1: } & \quad r=10 & \text { and } & T=3 \\
\text { Scheme 2: } & \quad r=10 & \text { and } & T=2 .
\end{array}
$$

For Scheme 1 , since $x_{r: n}<T$, the observed hybrid sample $\mathbf{x}=\left(x_{1: n}, \ldots, x_{d: n}\right)$ are 


$\begin{array}{lllllll}0.35 & 0.59 & 0.96 & 0.99 & 1.69 & 1.97 & 2.07 \\ 2.58 & 2.71 & 2.90 & & & & \end{array}$

In this case, $n=15, d=r=10$ and $T_{0}=\min \left\{x_{r: n}, T\right\}=2.90$. From the above sample, we obtain

$$
\sum_{i=1}^{d} x_{i}+(n-d) T_{0}=31.31
$$

which yields

$$
\widehat{\theta}=\frac{d}{\sum_{i=1}^{d} x_{i}+(n-d) T_{0}}=0.3194 .
$$

For Scheme 2, we have $x_{r: n}>T$, thus the observed hybrid sample are

$$
\begin{array}{llllll}
0.35 & 0.59 & 0.96 & 0.99 & 1.69 & 1.97 .
\end{array}
$$

In this case, $n=15, d=6$ and $T_{0}=\min \left\{x_{r: n}, T\right\}=2$. From the above sample, we obtain

$$
\sum_{i=1}^{d} x_{i}+(n-d) T_{0}=24.55
$$

which yields

$$
\widehat{\theta}=\frac{d}{\sum_{i=1}^{d} x_{i}+(n-d) T_{0}}=0.4073
$$

For each case, our aim is that to predict $Y=X_{s+d: n}(s=1,2, \ldots, n-d)$ based on observed data $\mathbf{x}=\left(x_{1: n}, \ldots, x_{d: n}\right)$. Using different methods discussed in Sections 3 and 4, we computed different point predictors and also the $95 \%$ PIs. The results are displayed in Table 1. Note that for computing Bayesian predictions, since we do not have any prior information, we assumed that the prior on $\theta$ is non-informative, i.e. $a=b=0$. 


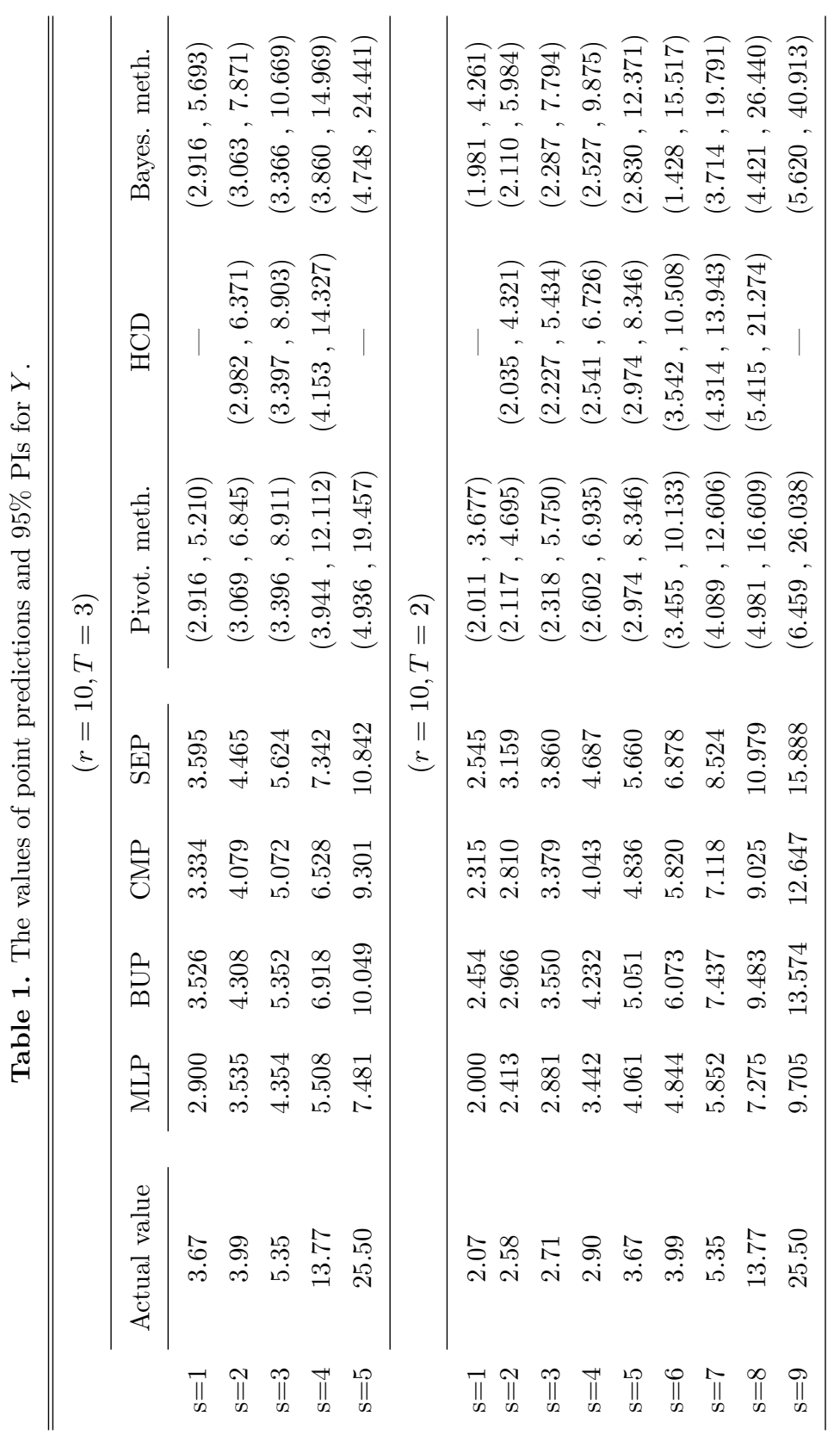




\section{Simulation and Discussion}

In this section, different prediction methods have been compared using a Monte Carlo simulation. We have generated hybrid censored data for different $n, r$, and $T$. The hybrid censored data can be easily generated. For a given $n, r$, and $T$, we first generate the Type-II censored sample $x_{1: n}, \ldots, x_{r: n}$. If $x_{r: n}<T$, then the above Type-II censored sample is also a hybrid censored sample. If $x_{r: n}>T$, then we find $d$, such that $x_{d: n}<T<x_{d+1: n}$. In this case, the hybrid censored sample is $x_{1: n}, \ldots, x_{d: n}$.

In our simulation, we considered $n=30$ and $T=1$. We also used two parameter values $\theta=1,2$. We have generated 1000 hybrid censored sample $x_{1: n}, \ldots, x_{d: n}$ from the exponential model (1) and then obtained different point and interval predictors for $Y=X_{s+d: n}(s=1,2, \ldots, n-d)$. We then compared the performances of the different point predictors MLP, BUP, CMP, and the Bayes predictor SEP in terms of biases, and mean square prediction errors (MSPEs).

For computing different Bayesian predictors, we assume two priors as follows:

$$
\begin{array}{lll}
\text { Prior 1: } & a=0, & b=0 . \\
\text { Prior 2: } & a=3, & b=3 .
\end{array}
$$

For various choices of $r$ and $s$, Table 2 presents the average biases, and MSPEs of different point predictors from this simulation study. All the computations are performed using Visual Maple (V16) package. For solving the nonlinear equations, we used the function fsolve from this package.

From Table 2, we observe that the BUP is the best predictor. The CMP is the second best predictor. We also observe that the MLP does not work well. Comparing the two Bayesian predictors based on two priors 1 and 2 clearly shows that the Bayesian predictors based on prior 2 perform better than the Bayesian predictors based on non-informative prior 1, in terms of both biases and MSPEs. The Bayes predictors based on both priors perform better than the MLPs.

We also compared different PIs in terms of the average confidence lengths, and coverage percentages. Table 3 presents the average confidence lengths and the corresponding coverage percentages. The nominal level for the confidence intervals is 0.95 in each case.

From Table 3, it is clear that the HCD method is the best procedure to obtain PI. It provides the shortest confidence length. The pivotal method 
is the second best procedure. Comparing the two Bayesian predictors based on two priors 1 and 2, we observe that the Bayesian predictors based on prior 2 perform better than the Bayesian predictors based on non-informative prior 1 , in term of the average confidence length. Bayesian PIs are wider than the non-Bayesian PIs and they provide the highest simulated coverage percentages.

From Tables 2 and 3, for fixed $r$, when $s$ is increasing the biases, MSPEs and the average confidence lengths are increasing which is reasonable since in this case we move away from the available censored sample.

One of the referees mentioned that Bayesian predictors may not be robust by changing the prior parameters $a$ and $b$. In Table 4 , we provided the value of point predictors for different values of $a$ and $b$. In Table 5 , we also provided the average confidence lengths and coverage percentages of different PIs for different $a$ and $b$. From Tables 4 and 5 , it is clear that the results are not changed significantly. So, Bayesian predictors are almost robust by changing the prior parameters $a$ and $b$.

It is also important to discuss the behavior of point predictors when $n$ and $r$ increase. Table 6 presents the MSPEs of point predictors for different values of $n$ and $r$. From this table we note that when $n$ and $r$ increase, the MSPEs decrease.

\section{Conclusions}

In this paper, we have considered different predictors of times to failure of units censored in a hybrid censored sample from exponential distribution under non-Bayesian and Bayesian approaches. We compared the performances of the different approaches by Monte Carlo simulations. We note that Bayesian predictors based on informative prior perform better than the Bayesian predictors based on non-informative prior. We also note that the BUP and CMP compare very well in terms of both biases and MSPEs. Comparing different PIs, it is observed that the HCD PIs provide the shortest confidence lengths. An important problem will be to extend these results for other hybrid censoring schemes such as progressive hybrid censoring scheme. This work is in progress and it will be reported later. 


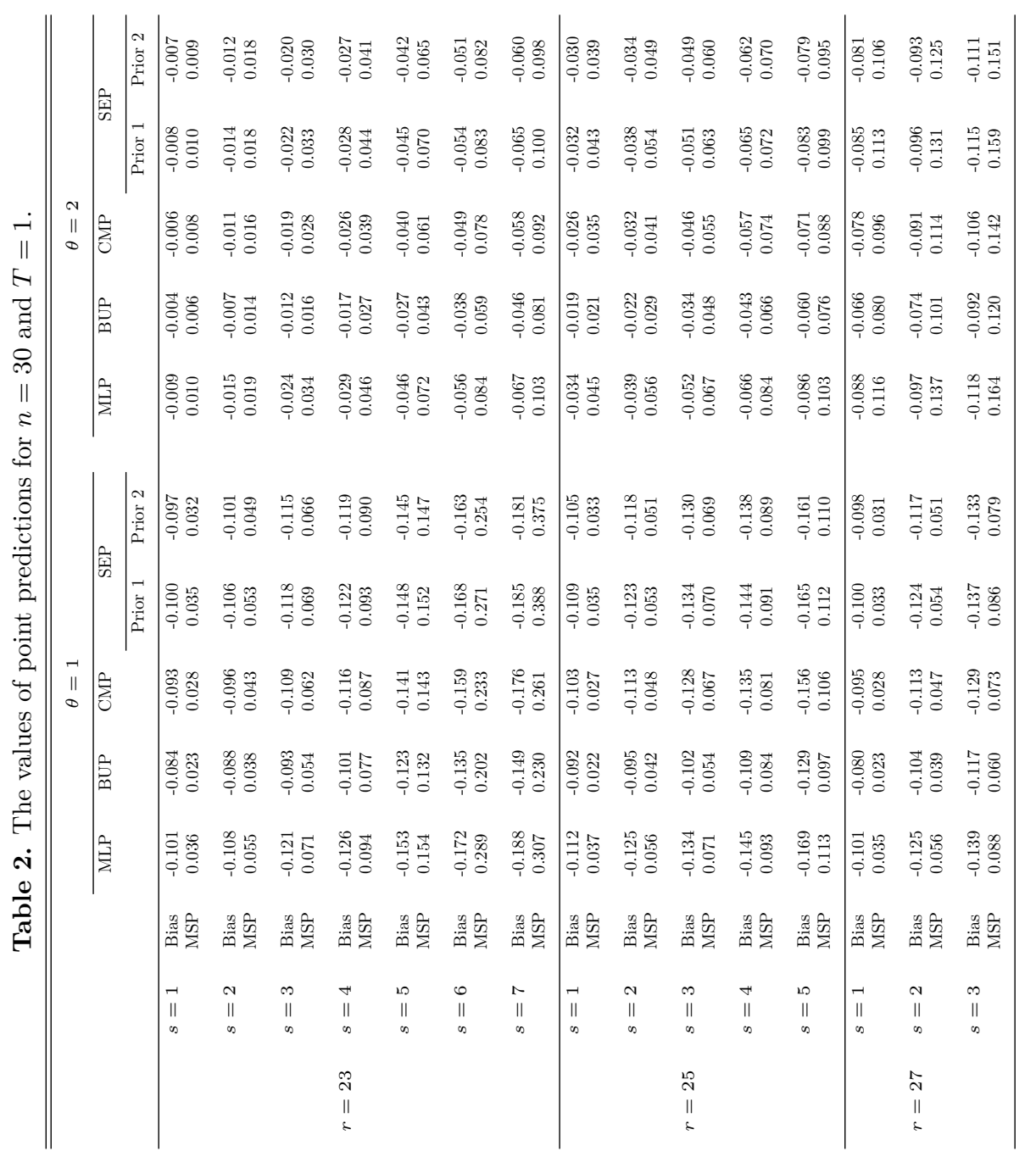




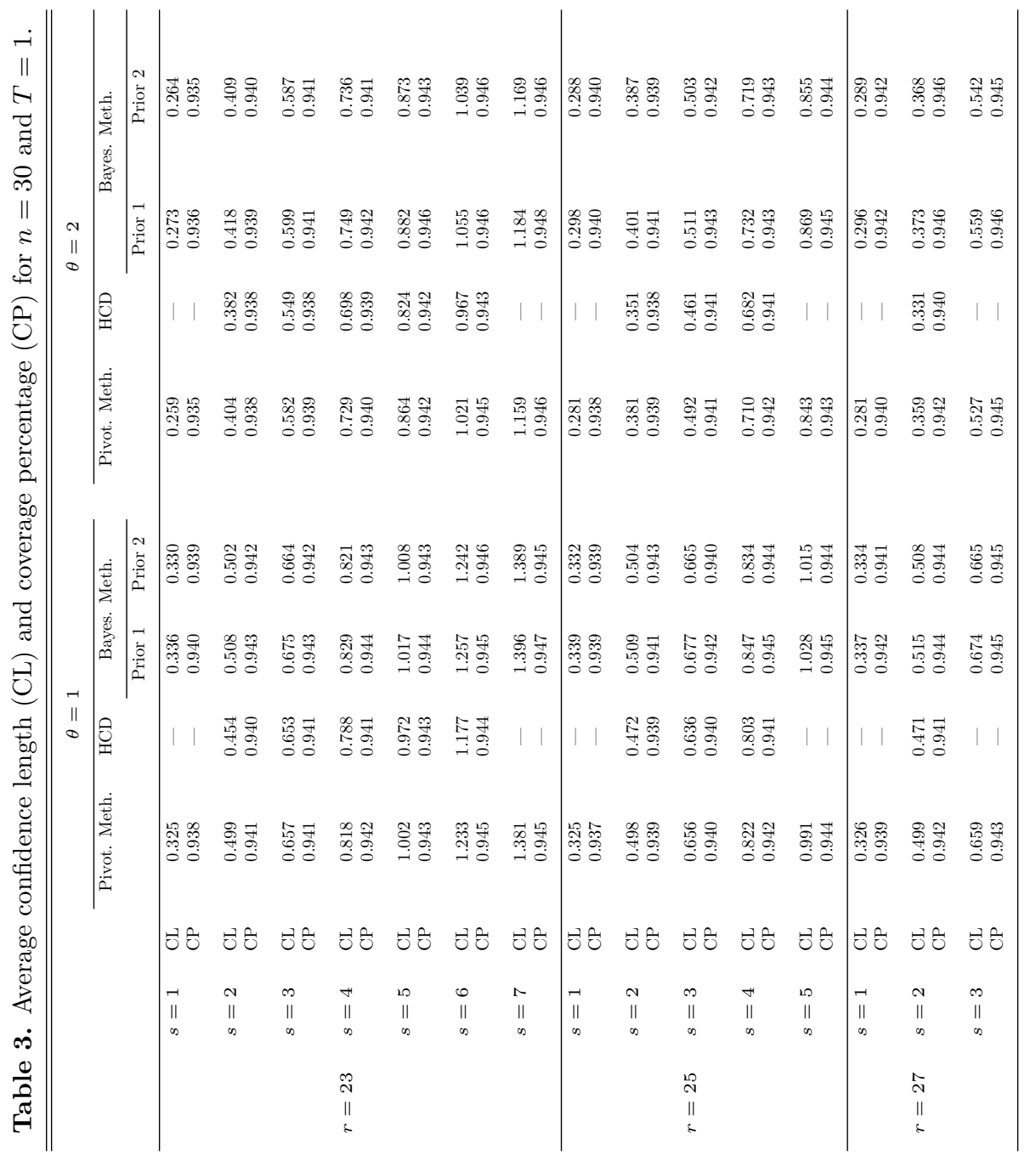




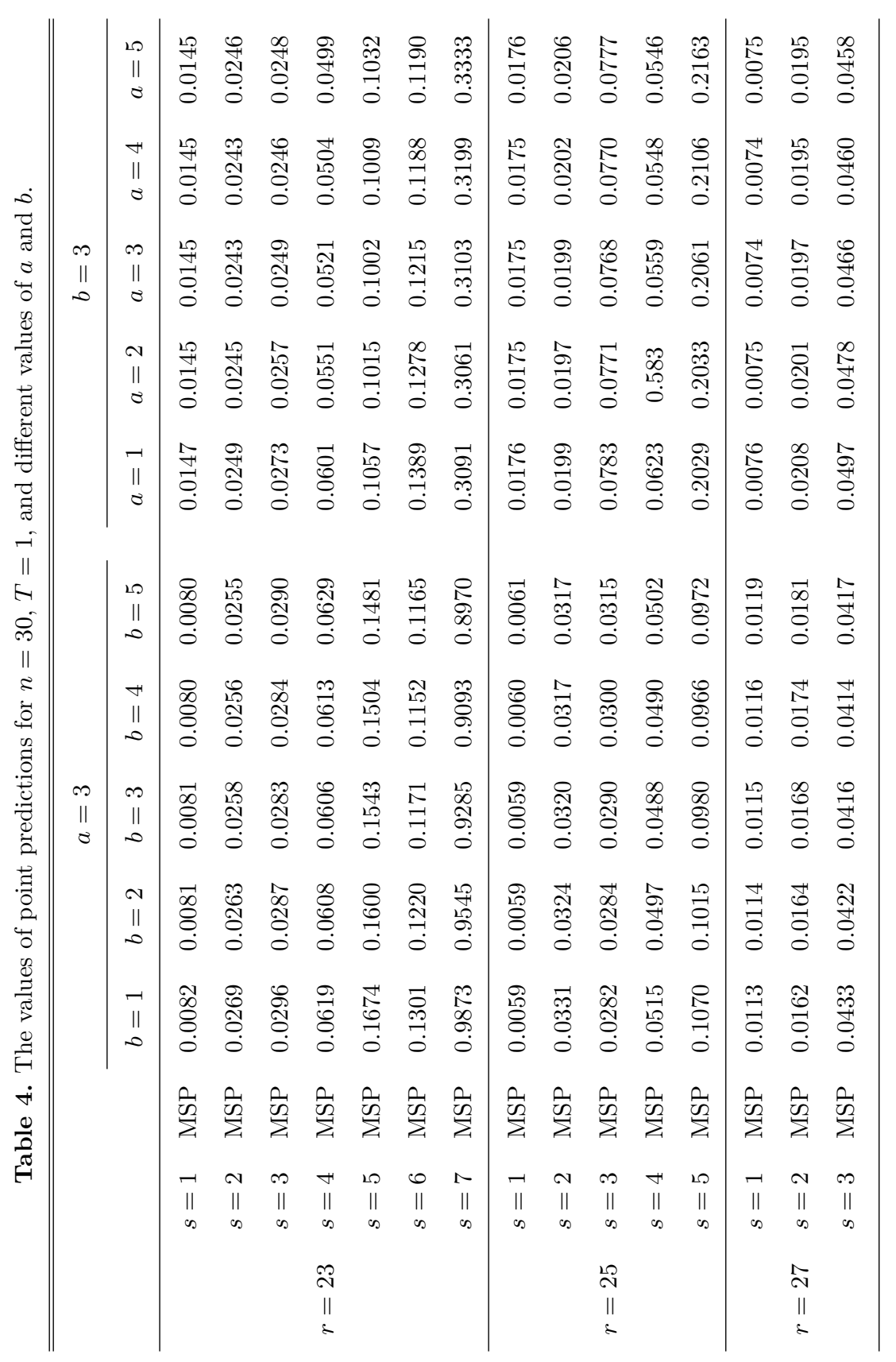




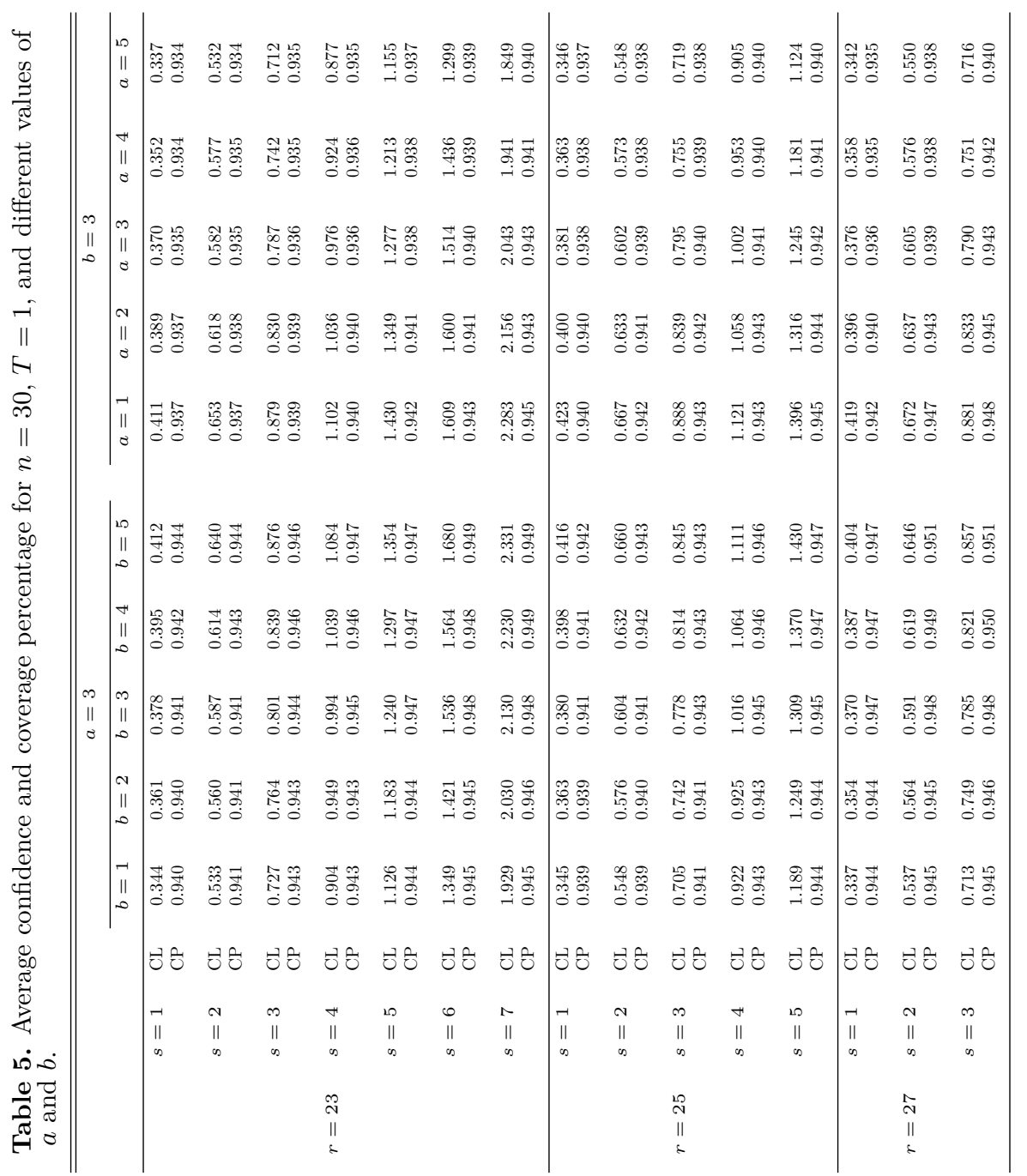




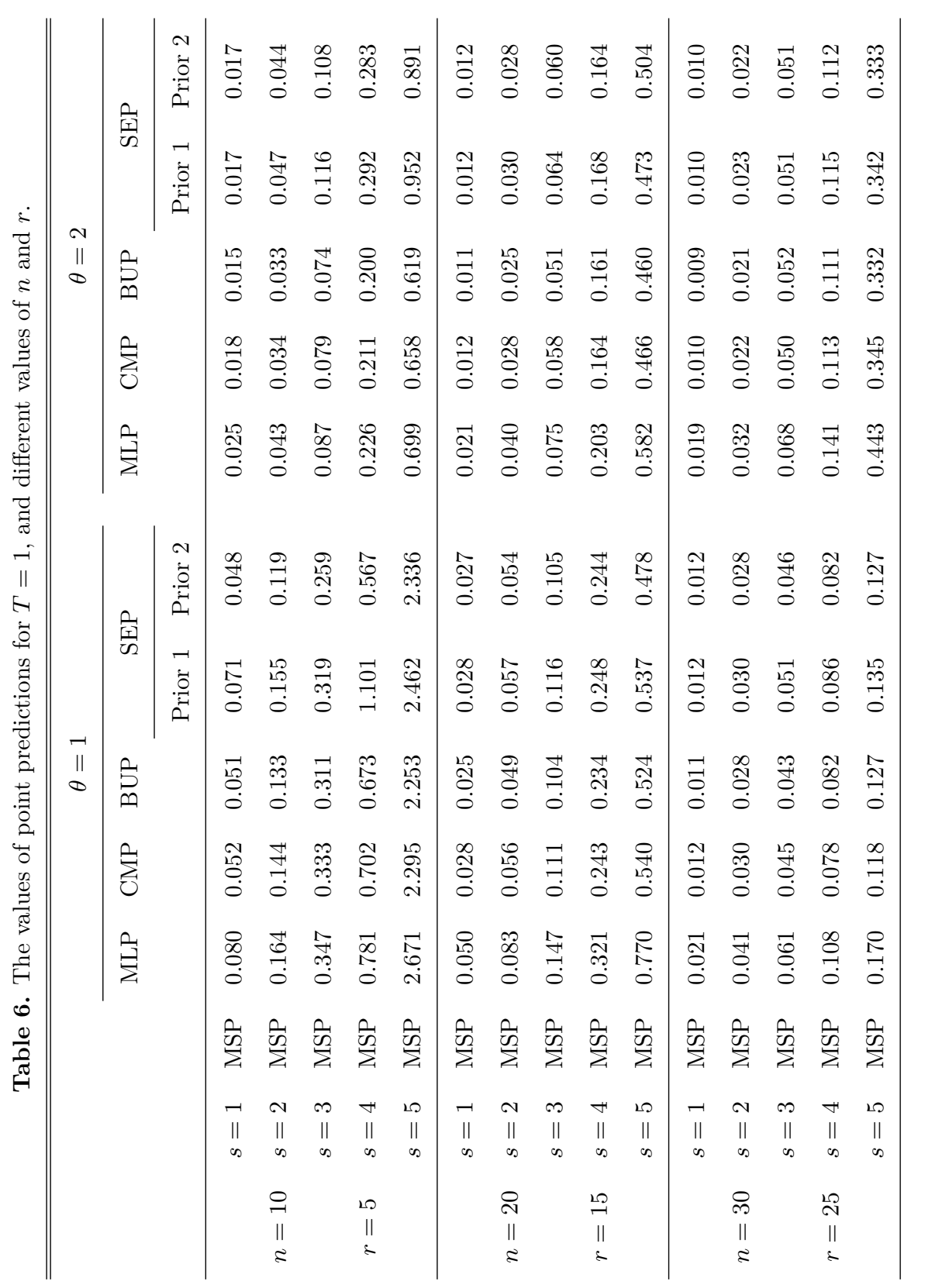




\section{Acknowledgements}

The authors would like to thank the referees for their careful reading of the paper and for their helpful comments in revising the paper. Research of the first author was supported by a grant from the University of Mazandaran.

\section{References}

Awad, A.M. and Raqab, M.Z. (2000). Prediction intervals for the future record values from exponential distribution: comparative study, Journal of Statistical Computation and Simulation, 65, 325-340.

Basak, I., Basak, P. and Balakrishnan, N. (2006). On some predictors of times to failures of censored items in progressively censored sample, Computational Statistics and Data Analysis, 50, 1313-1337.

Chen, S. and Bhattacharya, G.K. (1988). Exact confidence bounds for an exponential parameter under hybrid censoring, Communications in Statistics-Theory and Methods, 17, 18571870.

Childs, A., Chandrasekhar, B., Balakrishnan, N. and Kundu, D. (2003). Exact likelihood inference based on type-I and type-II hybrid censored samples from the exponential distribution, Annals of the Institute of Statistical Mathematics, 55, 319-330.

Draper, N. and Guttman, I. (1987). Bayesian analysis of hybrid life tests with exponential failure times, Annals of the Institute of Statistical Mathematics, 39, 219-225.

Dunsmore, I.R. (1983). The future occurrence of records. Annals of the Institute of Statistical Mathematics, 35, 267-277.

Ebrahimi, N. (1986). Estimating the parameter of an exponential distribution from hybrid life test, Journal of Statistical Planning and Inference, 14, 255-261.

Ebrahimi, N. (1992). Prediction intervals for future failures in exponential distribution under hybrid censoring, IEEE Transcation Reliability, 41, 127-132.

Epstein, B. (1954). Life tests in the exponential case, Annals of Mathematical Statistics, 25, $555-564$.

Fairbanks, K., Madison, R. and Dykstra, R. (1982). A confidence interval for an exponential parameter from a hybrid life test, Journal of the American Statistical Association, 77, 137140.

Gupta, R.D. and Kundu, D. (1998). Hybrid censoring schemes with exponential failure distribution, Communications in Statistics-Theory and Methods, 27, 3065-3083. 
Jeong, H.S., Park, J.I. and Yum, B.J. (1996). Development of $(r, T)$ hybrid sampling plans for exponential lifetime distributions, Journal of Applied Statistics, 23, 601-607.

Kaminsky, K.S. and Nelson, P.I. (1998). Prediction of Order Statistics. In: Balakrishnan, N., Rao, C.R. (Eds.), Handbook of Statistics, Order Statistics: Applications, vol. 17. NorthHolland, Amsterdam, pp. 431-450.

Kaminsky, K.S. and Rhodin, L.S. (1985). Maximum likelihood prediction, Annals of the Institute of Statistical Mathematics, 37, 707-717.

Kundu, D. (2007). On hybrid censoring Weibull distribution, Journal of Statistical Planning and Inference, 137, 2127-2142.

Kundu, D. and Banerjee, A. (2008). Inference based on Type-II hybrid censored data from a Weibull distribution, IEEE Transcation on Reliability, 57, 369-378.

Kundu, D. and Howlader, H. (2010). Bayesian inference and prediction of the inverse Exponential distribution for Type-II censored data, Computational Statistics and Data Analysis, 54, 1547-1558.

Lawless, I.L. (1971). A prediction problem concerning samples from the exponential distribution with applications in life testing, Technometncs, 13, 725-730.

Nelson, W.B. (1970). Statistical Methods for Accelerated Life Test Data-the Inverse Power Law Model. General Electric Co. Tech. Rep. 71-C011. Schenectady, New York.

Raqab, M.Z. and Nagaraja, H.N. (1995). On some predictors of future order statistics, Metron, 53, 185-204.

Ren, C., Sun, D. and Dey, K.D. (2006). Bayes and frequentist estimation and prediction for exponential distribution, Journal of Statistical Planning and Inference, 136, 2873-2897.

Tiku, M.L. and Akkaya, A.D. (2004). Robust Estimation and Hypothesis Testing, New Age International Limited Publishers, New Delhi.

\section{A. Asgharzadeh}

Department of Statistics,

University of Mazandaran,

Babolsar, Iran.

email: A.Asgharzadeh@umz.ac.ir

\section{R. Valiollahi}

Department of Statistics, Semnan University, Semnan, Iran. 